\title{
Educação sexual na prática pedagógica de professores da rede básica de ensino
}

\author{
Sex education in the pedagogical practice of public school teachers
}

Educación sexual en la práctica pedagógica de profesores de la red básica de enseñanza

Camila Borges Rufino ${ }^{1}$, Laurena Moreira Pires ${ }^{2}$, Patrícia Carvalho Oliveira ${ }^{3}$, Sandra Maria Brunini Souza ${ }^{4}$, Márcia Maria de Souza ${ }^{5}$

${ }^{1}$ Enfermeira. Goiânia, GO, Brasil. E-mail: camilabrufino@gmail.com.

${ }^{2}$ Enfermeira. Discente do Programa de Pós-Graduação em Enfermagem, nível Mestrado, da Faculdade de Enfermagem da Universidade Federal de Goiás (FEN/UFG). Goiânia, GO, Brasil. E-mail: laurena 89@hotmail.com.

${ }^{3}$ Enfermeira, Mestre em Enfermagem. Goiânia, GO, Brasil. E-mail: patico.nurse@gmail.com.

${ }^{4}$ Enfermeira, Doutora em Enfermagem. Professora Adjunta da FEN/UFG. Goiânia, GO, Brasil. E-mail: sandrabrunini@gmail.com.

${ }^{5}$ Enfermeira, Doutora em Ciências da Saúde. Professora Adjunta da FEN/UFG. Goiânia, GO, Brasil. E-mail: marcia.fen@gmail.com.

\section{RESUMO}

Estudo descritivo realizado com 29 professores de três escolas públicas estaduais em Goiânia, Goiás, que responderam a um questionário semiestruturado cujo objetivo foi verificar a prática pedagógica em educação sexual bem como as dificuldades na temática e necessidades de capacitação. A maioria tem entre 25 e 35 anos (74\%) e é do sexo masculino (69\%). A metade possui pós-graduação (54\%) e é da área de ciências humanas (49\%). Quase a totalidade tem dificuldade em trabalhar a temática (89\%) e necessitam se capacitar (93\%). Conteúdos sobre sexualidade não constavam nos Projetos Políticos Pedagógicos (76\%) e a disciplina biologia apontada para o ensino da temática (55\%), realidade que contradiz os Parâmetros Curriculares Nacionais (PCN), que se pauta pela transversalidade. Há necessidade de parcerias entre a saúde, especialmente a Estratégia Saúde da Família (ESF), e a educação, como a Instituição de Ensino Superior, como suporte didático-pedagógico aos professores da rede básica de ensino para o trabalho em sexualidade.

Descritores: Sexualidade; Instituições Acadêmicas; Enfermagem; Saúde Pública.

\section{ABSTRACT}

This descriptive study was performed with 29 teachers from three public state schools in Goiânia, Goiás state. The teachers answered a semi-structured questionnaire that aimed at verifying the pedagogical practice in sex education, difficulties related to the theme, and the need for training. Most teachers were male (69\%) and aged between 25 and 35 years (74\%). Half held a graduate degree (54\%) in human sciences (49\%). Nearly all teachers find difficulties in working the topic (89\%) and need training (93\%). Contents on sexuality were not addressed in the Political Pedagogical Projects (76\%) and the biology program aimed at teaching the theme (55\%), a reality that disagrees with the National Curricular Standards, which is based on transversality. Partnerships between health and education must be established, particularly between the Family Health Strategy the higher education institutions, providing public school teachers with the necessary didactic-pedagogical support to address sexuality in the classroom.

Descriptors: Sexuality; Schools; Nursing; Public Health.

\section{RESUMEN}

Estudio descriptivo realizado con 29 profesores de tres escuelas públicas estatales en Goiânia-Goiás, que respondieron cuestionario semiestructurado, objetivando verificar la práctica pedagógica en educación sexual, las dificultades en la temática y necesidad de capacitación. La mayoría tiene entre 25 y 35 años (75\%), sexo masculino (69\%). La mitad posee posgraduación (54\%) y pertenece al área de Ciencias Humanas (49\%). Casi la totalidad tiene dificultades en trabajar la temática (89\%) y necesitan capacitarse (93\%). No constaban contenidos sobre sexualidad en los Proyectos Políticos Pedagógicos (76\%) y la disciplina biología, señalada para enseñanza de la temática (55\%), realidad que contradice los Parámetros Curriculares Nacionales (PCN) que determinan la transversalidad. Existe necesidad de alianzas entre la salud, especialmente la Estrategia Salud de la Familia (ESF) y educación, como la Institución de Enseñanza Superior, como soporte didáctico-pedagógico a profesores de la red básica de enseñanza para el trabajo en sexualidad.

Descriptores: Sexualidad; Instituciones Académicas; Enfermería; Salud Pública. 


\section{INTRODUÇÃO}

A educação é uma área de fundamental importância para a sociedade e foco de discussão em importantes conferências realizadas em todo o mundo. Como parte da educação formal, a educação sexual deve permear discussões sobre a sexualidade no contexto escolar, devendo transcender a visão tecnicista e biologicista ainda bastante presente na vida das pessoas e nos currículos escolares ${ }^{(1)}$.

A sexualidade está presente na vida das pessoas, agrega aspectos individuais, sociais e culturais pautadas em histórias, mitos, símbolos e experiências advindas da própria infância, e se exacerba principalmente, na fase da adolescência na socialização com amigos, família, mídia e escola ${ }^{(2)}$.

A propagação e constância na mídia sobre cenas de erotismo e a banalização do sexo que podem resultar na precocidade da iniciação sexual traz repercussões pessoais e sociais importantes, pois analisando seus comportamentos estes estão, geralmente, em buscas de aventura e satisfação imbuídas de sentimentos intensos como desejos e paixões que podem deixá-los expostos à situações de vulnerabilidade. Estudos realizados com professores mostrou sobre a necessidade de promover intervenções no campo da promoção da saúde sexual e reprodutiva com escolares, considerando que a sexualidade envolve dimensões variadas de gênero, identidade sexual, reprodução, prazer, envolvimento emocional, prevenção e orientação sexual(2-3).

Temáticas que envolvem a sexualidade vêm sendo discutidas na educação básica (ensinos fundamental e médio) desde a década de 20 , mas a inclusão nos currículos de $1^{\circ}$ e $2^{\circ}$ graus se intensificou a partir da década de 70 que por determinação da lei que regulou as Diretrizes e Bases da Educação Brasileira passa a obrigatoriedade de incluir programas de saúde e conteúdos relacionados à sexualidade humana no currículo escolar o que não era oficialmente reconhecido e permitido(2).

Foi somente a partir de 1980, sobretudo, com o advento da Síndrome da Imunodeficiência Adquirida (AIDS) e mudanças comportamentais, que a demanda por estudos envolvendo a temática sexualidade nas instituições de ensino aumentou, devido na época, ao intenso crescimento da gravidez entre adolescentes e a grande preocupação de pesquisadores e educadores em relação ao fenômeno da AIDS na população jovem, fenômeno conhecido como juvenilização da Aids ${ }^{(4-5)}$. Por isso é necessário que discussões sejam suscitadas em todo o ambiente em que o escolar esteja inserido, seja na família, na socialização com os amigos e na instituição de ensino em que estuda.

No Brasil, de 1980 a 2011 foram notificados 608.230 casos de HIV/Aids, sendo que $11 \%$ (66.698) entre jovens de 15 a 24 anos $^{(5)}$. Nesse contexto é necessário promover a instrumentalização de profissionais tanto da saúde quanto da educação na perspectiva da redução das vulnerabilidades às DST entre os jovens, em qualquer ambiente que frequenta. No contexto educacional, a escola é considerada espaço potencial para socialização e troca de experiências e também reconhecida para trabalhar temas comportamentais.

Estudos mostram que o conhecimento sobre sexualidade entre os adolescentes é limitado, e muitas vezes incompleto, muitas vezes apreendido na socialização com os amigos, em família, na comunidade, com profissionais de saúde e na escola, mas nem sempre estas informações são verdadeiras, eficazes e impactantes ${ }^{(6-7)}$.

Como estimulo e rede de apoio aos escolares, a escola é responsável pela organização e repasse de informações de todas as áreas do conhecimento além de ser o espaço coletivo apropriado para a socialização, exercício da autonomia, identificação grupal e troca de experiências $^{(8-9)}$, além de extensão da família deve ser também promotora da educação sexual, especialmente porque o professor é considerado o profissional que tem maior proximidade e acesso aos alunos e reconhecido como grande aliado e conselheiro no espaço escolar ${ }^{(3,6)}$.

Considerando que é de responsabilidade do sistema escolar promover a educação integral da criança e adolesente é necessário que se discuta a sexualidade por meio da educação sexual de forma integral e multidisciplinar, pautado na transversalidade como já proposto pelo Parâmetro Curricular Nacional (PCN) $)^{(10)}$.

O PCN foi proposto em 1996 com intuito de incluir nos Projetos Políticos Pedagógicos (PPP) temas na área da sexualidade a serem trabalhados de forma transversal, ou seja, em todas as áreas do conhecimento, na abordagem e aprofundamento necessário, considerando as necessidades e interesses dos escolares. Sobretudo a transversalidade implica na necessidade do 
professor não apenas de dominar o conteúdo da sua matéria específica, mas conhecer outros conteúdos de diferentes áreas do conhecimento e interagir com a realidade dos alunos, pois estes ainda tem apresentado conhecimentos errôneos e deficientes em temas específicos envolvendo a sexualidade(11). Assim faz-se necessário discussões e reflexões críticas de modo a proporcionar a participação tanto de professores quanto dos alunos, especialmente incentivando o protagonismo juvenil(8).

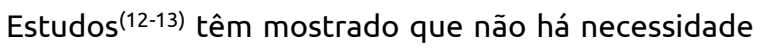
que o professor seja um especialista em educação sexual, mas um profissional que mobilize o ambiente escolar e com capacidade de articular conteúdos e criar contextos pedagógicos adequados para reflexões e debates de ideias. Este profissional deve estar em constante processo de atualização de seus conhecimentos, por meio de educação continuada de forma a ensinar o outro a pensar e discutir, método principal no processo ensino aprendizagem devendo estar presente nos diferentes níveis educacionais.

Mas sabemos que, de fato, os professores não recebem informações suficientes em sexualidade na sua formação, por isso trabalhar conteúdos que envolvem esta temática e suas especificidades no ambiente escolar é considerado tema delicado e difícil de ser trabalhado. De acordo com pesquisas realizadas há envolvimentos diretos com questões socioculturais e até religiosos como sentimentos, culturas, valores, tabus e crenças, ainda bastante arraigados na cultura brasileira ${ }^{(2,13)}$.

Como estratégia de apoiar as instituições de ensino no contexto escolar, na área da sexualidade, em 2006 e 2008 o governo federal apresentou, respectivamente, o projeto SPE (Saúde e Prevenção nas Escolas) e o programa PSE (Programa Saúde na Escola) com propostas semelhantes, dentre outras ações, de subsidiar e incentivar cursos de capacitação para professores no âmbito da saúde sexual e reprodutiva no sentido de minimizar as diversas vulnerabilidades a que os jovens estão expostos com foco na prevenção das doenças transmitidas pela via sexual como Aids, hepatites, HPV, dentre outras e, sobretudo, gravidez precoce ${ }^{(14-15)}$.

Recentemente em âmbito nacional foi realizada a Pesquisa Nacional de Saúde dos Escolares (PeNSE) ${ }^{(16)}$ que visou conhecer a saúde geral da população de escolares, especialmente os fatores de risco e proteção. Dentre os vários resultados obtidos na pesquisa outro estudo(17) mostrou as situações relacionadas à saúde sexual dos adolescentes e as vulnerabilidades que os escolares estão expostos. Dentre os resultados encontrados está a precocidade da iniciação sexual tanto para meninos quanto para meninas, evidenciando, portanto sobre a necessidade de promover orientações e apoio por parte dos familiares, profissionais da saúde e de educação nesta fase da vida para que tenham uma saúde sexual mais saudável, prazerosa e segura(18).

Considerando que a saúde e a educação estão diretamente interligadas e são dois pilares da sobrevivência humana os referidos projetos e a pesquisa realizada tem sido amplamente divulgada e reconhecida para subsidiar trabalhos de professores em abordagens no âmbito da prevenção da saúde sexual e reprodutiva em todas as escolas brasileiras.

Como justificativa este estudo se propôs conhecer as práticas pedagógicas dos professores no trabalho a educação sexual, suas deficiências e necessidades para assim planejar, conjuntamente com a gestão escolar local, ações futuras mais assertivas e seguras por meio da educação continuada e instrumentalização dos professores com conteúdos técnicos científicos atualizados, e metodologias mais participativas e vivenciais. Vislumbramos a possibilidade de realização de trabalhos e apoio por parte das Instituições de Ensino Superior (IES) com projetos de pesquisa e extensão no sentido de ofertar ações como educação continuada e educação permanente aos professores de outros níveis de ensino, educação básica (ensinos fundamental e médio), reconhecido como importante papel social da Universidade.

Mediante ao exposto, este estudo objetivou verificar a prática pedagógica em educação sexual de professores de instituições da rede básica de ensino, bem como conhecer as dificuldades na temática e necessidades de capacitação.

\section{MATERIAL E MÉTODOS}

Trata-se de um estudo descritivo realizado em três instituições da rede básica de ensino da rede estadual, localizadas na região leste do município de Goiânia-GO. As escolas foram selecionadas por ter cobertura de $100 \%$ da Estratégia Saúde da Família (ESF), implantação 
prévia do SPE e PSE, aceitação dos diretores/coordenadores locais em participar do estudo e permissão de desenvolvimento da pesquisa somente com professores do ensino médio.

A amostra foi composta por 29 professores que aceitaram participar do estudo de um total de 35 que desenvolvem trabalhos exclusivamente no ensino médio, com alunos na faixa etária de 12 a 24 anos (períodos matutino e noturno). O período vespertino é destinado ao ensino fundamental com composição de outro grupo de professores.

Os dados foram coletados no período de novembro de 2010 a janeiro de 2011, com datas pré-agendadas pactuadas com os professores. Рara a coleta de dados foi utilizado um questionário semiestruturado, contendo questões abertas e fechadas, autoaplicável contendo perguntas sobre: características sociodemográficas: idade, sexo, estado civil e filhos; características profissionais: tempo de formação, área de atuação, possui Pós-Graduação, tempo de trabalho na instituição e carga horária de trabalho e características da prática pedagógica: experiência na área da sexualidade, dificuldades e necessidades dos professores no trabalho sobre sexualidade. $\mathrm{O}$ instrumento de coleta de dados foi avaliado previamente, por meio de teste piloto realizado com professores de outra instituição com características semelhantes as do estudo, localizada em outra região do município, que verificou sua objetividade, clareza e pertinência em relação aos objetivos propostos.
Os dados foram organizados na planilha Excel. A análise estatística constituiu-se pela identificação das variáveis e apresentados por meio de frequências e percentagens, utilizando o programa estatístico Epi-info versão 6.04 for Windows, desenvolvido pelo CDC (Centers for Diseases Control and Prevention), EUA.

Este estudo foi aprovado pelo Comitê de Pesquisa Médica Humana e Animal do Hospital das Clínicas da Universidade Federal de Goiás, sob o protocolo $n^{\circ}$ 058/2009, sob o cumprimento de todos os procedimentos metodológicos da resolução 196/96 do Conselho Nacional de Saúde (CNS) do Ministério da Saúde, e teve apoio financeiro da Fundação de Amparo a Pesquisa Goiana - FAPEG (Nº01/2008).

\section{RESULTADOS}

Participaram do estudo 29 professores. A maioria dos participantes era do sexo masculino $(69,0 \%)$ e $31,0 \%$ do sexo feminino, $74,0 \%$ possuía idade entre 25 e 35 anos e $26,0 \%$ tinham entre 40 e 60 anos. Do total, $62,0 \%$ eram casados e $72,0 \%$ possuíam de um a dois filhos.

A Tabela 1 apresenta as características profissionais da população estudada. Todos os professores possuem tempo de formação superior a um ano. Observa-se uma maior prevalência de professores com formação na área das ciências humanas (49\%) e com pelo menos uma PG (54,0\%). Em relação ao tempo de trabalho na instituição $45,0 \%$ informaram trabalhar há pelo menos um ano e a metade $(51,0 \%)$ cumpre carga horária superior a 30 horas semanais.

Tabela 1: Características profissionais dos professores ( $n=29)$. Goiânia, GO, Brasil, 2010.

\begin{tabular}{|c|c|c|}
\hline Características & $\mathbf{N}$ & $\%$ \\
\hline \multicolumn{3}{|l|}{ Tempo de formação } \\
\hline 1 a 6 anos & 14 & 48 \\
\hline 7 a 16 anos & 8 & 28 \\
\hline 17 a 26 anos & 7 & 24 \\
\hline \multicolumn{3}{|l|}{ Área de formação } \\
\hline Ciências Biológicas & 7 & 24 \\
\hline Ciências Exatas & 8 & 27 \\
\hline Ciências Humanas & 14 & 49 \\
\hline \multicolumn{3}{|l|}{ Pós-graduação } \\
\hline Uma & 15 & 54 \\
\hline Duas ou três & 4 & 14 \\
\hline Nenhuma & 9 & 32 \\
\hline \multicolumn{3}{|l|}{ SI $(n=1)$} \\
\hline \multicolumn{3}{|l|}{ Tempo de trabalho na instituição } \\
\hline Menos de 1 mês & 2 & 7 \\
\hline 1 mês a 1 ano & 13 & 45 \\
\hline 2 a 10 anos & 7 & 24 \\
\hline 16 a 25 anos & 7 & 24 \\
\hline \multicolumn{3}{|l|}{ Carga horária de trabalho na instituição } \\
\hline 10 a 20 horas/aula & 6 & 21 \\
\hline
\end{tabular}


A caracterização dos alunos em relação a temática sexualidade, na opinião dos professores está descrita na

Tabela 2.

Tabela 2: Interesse e conhecimento dos alunos, segundo os professores (n=29). Goiânia, GO, Brasil, 2010.

\begin{tabular}{|c|c|c|}
\hline Características & $\mathbf{N}$ & $\%$ \\
\hline \multicolumn{3}{|l|}{ Interesse pela temática sexualidade } \\
\hline Sim & 28 & 96,5 \\
\hline Não & 1 & 3,5 \\
\hline \multicolumn{3}{|l|}{ Como foi o interesse? } \\
\hline Perguntas ao professor & 15 & 52 \\
\hline Discussão/debates em sala de aula & 10 & 34,4 \\
\hline $\begin{array}{l}\text { Conversas entre os alunos } \\
\text { Conhecimento em DST }\end{array}$ & 4 & 13,6 \\
\hline $\operatorname{Sim}$ & 25 & 86,2 \\
\hline Não & 4 & 13,8 \\
\hline
\end{tabular}

O interesse dos alunos pela temática sexualidade foi citado por $96 \%$ dos professores, sendo que a metade (52\%) relatou sob a forma de perguntas ao professor, seguido de discussão e debates em sala de aula. A maioria (86\%) dos professores informou que os alunos conhecem sobre prevenção, DST e conteúdos referentes à temática sexualidade.

A Tabela 3 mostra as práticas pedagógicas, dificuldades e necessidades dos professores em relação ao trabalho da temática sexualidade no contexto escolar.

Tabela 3: Caracterização da prática pedagógica, dificuldade e necessidade dos professores na abordagem da temática sexualidade ( $n=29)$, Goiânia-GO, 2010

\begin{tabular}{|c|c|c|}
\hline Características & $\mathbf{N}$ & $\%$ \\
\hline \multicolumn{3}{|l|}{ Experiência na temática } \\
\hline Sim & 4 & 13,8 \\
\hline Não & 25 & 86,2 \\
\hline \multicolumn{3}{|l|}{ Receberam capacitação } \\
\hline Sim & 8 & 27,6 \\
\hline Não & 21 & 72,4 \\
\hline \multicolumn{3}{|l|}{ Presença da temática sexualidade no Projeto Político Pedagógico } \\
\hline Sim & 7 & 24,1 \\
\hline Não & 22 & 75,9 \\
\hline \multicolumn{3}{|l|}{ Dificuldade na abordagem } \\
\hline Sim & 26 & 89,6 \\
\hline Não & 3 & 10,4 \\
\hline \multicolumn{3}{|l|}{ Necessidade de Capacitação } \\
\hline Sim & 27 & 93,1 \\
\hline Não & 2 & 6,9 \\
\hline \multicolumn{3}{|l|}{ Sugestão para atualização } \\
\hline Educação continuada & 26 & 89,6 \\
\hline Uso da internet, EAD & 3 & 10,4 \\
\hline \multicolumn{3}{|l|}{ Métodos e Recursos para capacitação } \\
\hline Tempo e Planejamento & 12 & 41,4 \\
\hline Linguagem correta & 5 & 17,2 \\
\hline Material didático & 10 & 34,5 \\
\hline Desinteresse em capacitação & 2 & 6,9 \\
\hline \multicolumn{3}{|l|}{ Disciplinas que devem abordar a temática } \\
\hline Todas as disciplinas & 2 & 6,9 \\
\hline Biologia & 16 & 55,2 \\
\hline Português & 4 & 13,8 \\
\hline Matemática & 3 & 10,3 \\
\hline Geografia & 2 & 6,9 \\
\hline Educação Física & 2 & 6,9 \\
\hline
\end{tabular}

Do total de entrevistados $86,2 \%$ referiram não possuir formação na temática sexualidade. Quando questionados se conteúdos sobre sexualidade estão contemplados no PPP, 75,9\% dos professores disseram que não. Proporções semelhantes foram encontradas nas variáveis sobre dificuldade $(89,6 \%)$ e necessidade de capacitações $(93,1 \%)$, sob a forma de educação continuada $(89,6 \%)$.

Em relação a métodos e recursos para capacitações citaram que necessitam de tempo e planejamento para 
se capacitarem, assim como materiais didáticos. Quando indagados sobre quais disciplinas devem trabalhar conteúdos que contemple a área da sexualidade, a metade dos professores $(55,2 \%)$ citou que seria biologia seguida de disciplinas da área de humanas e exatas, seguido de português (13,8\%), geografia $(6,9 \%)$ e matemática (10,3\%), respectivamente. Dados apresentados na Tabela 3.

\section{DISCUSSÃO}

Mais da metade dos participantes do estudo $(72,0 \%)$ possuem um ou dois filhos, e a maioria destes com filhos entre 11 e 20 anos, fase da adolescência, dado não apresentado anteriormente. De acordo com o Estatuto da Criança e do Adolescente (ECA) esta fase está compreendida entre 12 e 18 anos $^{(19)}$. Há um quantitativo muito grande de adolescentes nesta fase inseridos nas instituições de ensino, sendo que cada adolescente chega à instituição com caraterísticas semelhantes, apresentando uma série de transformações, descobertas e vivências, permeadas por sentimentos, emoções e desejos, próprios desta fase ${ }^{(9,18)}$. Os professores com filhos na fase da adolescência que relacionam diariamente com seus alunos na mesma faixa etária necessitam estar mais seguros nas abordagens de temas comportamentais, em especial relacionados à sexualidade, pois o exercício de convivência e confiança mútua certamente contribuirá para a formação saudável tanto de seus alunos quanto de seus filhos.

A população jovem está propensa a situações de vulnerabilidades que podem modificar suas vidas e definir seus futuros. Assim o fenômeno da sexualidade, incluindo as DST/HIV/AIDS e gravidez não planejada constituem problemáticas ainda bastante comuns nas instituições escolares brasileiras.

A sexualidade no contexto escolar é tema de grande interesse e impacto para os jovens, além de ser reconhecido como assunto emergente e de grande importância, devendo ser incorporado nos currículos de $1^{\circ}$ e $2^{\circ}$ graus das instituições de ensino(10,12).

Praticamente a metade dos professores possui PósGraduação, sugerindo um bom nível de escolaridade. Um estudo mostrou que embora os profissionais possuam cursos de pós-graduação, estes podem não ser em suas áreas específicas de formação, podendo dificultar suas práticas pedagógicas ${ }^{(4)}$.
A área de formação predominante foi a de ciências humanas. De acordo com este dado, é possível compreender que os professores pesquisados, por serem da área de ciências humanas possivelmente não trabalhem ou não foram despertados para incluírem temas transversais como a educação sexual em seus conteúdos programáticos, conforme preconizado pelo PNC(10). Ainda é comum encontrar estudos que mostrem que a educação sexual deve ser trabalhada fundamentalmente nas disciplinas biologia, ciências e religião(1,3), desconsiderando a necessidade de discussão nos aspectos e influências sociais, históricas e culturais da sexualidade humana, necessitando, portanto de "desbiologizar" a temática ${ }^{(13,20)}$. Esta informação ratifica o resultado dos professores quando perguntado sobre quais disciplinas devem abordar a temática sexualidade e confirmada como sendo a disciplina de biologia.

Foi identificado nesta investigação que quase a totalidade dos alunos apresentou interesse na temática sexualidade. Os professores devem aproveitar este interesse e trabalhar conteúdos relativos às questões comportamentais, contextualizando, portanto, as experiências e vivências emergidas de suas práticas com conteúdos das aulas, pois os adolescentes estando em grupos favorece à socialização. O professor é um agente mobilizador e referência para o aluno, que sentem no professor segurança para falar sobre suas experiências e expor suas dúvidas e, talvez não tenham coragem e liberdade ao diálogo com os pais ou outros membros da família(21).

Ainda nesta perspectiva, estudos têm mostrado que os adolescentes estão iniciando suas atividades sexuais cada vez mais precocemente ${ }^{(17,20)}$. Analisando o aspecto do interesse dos alunos neste estudo e a precocidade da iniciação sexual, torna-se necessário que discussões sejam suscitadas no ambiente escolar, pois de acordo com pesquisas realizadas(20-21), o jovem que geralmente está em busca de situações novas e satisfações elegem o sexo como fonte de bem-estar aumentando, por exemplo, o risco para aquisição de doenças transmitidas sexualmente.

De acordo com os resultados apresentados neste estudo os professores não possuem experiência na temática, não receberam capacitações, a temática não está presente nos PPP, relataram sentir dificuldades com o tema e necessidade de se capacitar. 
Embora a inclusão da Educação Sexual como tema transversal proposto pelo $\mathrm{PCN}^{(10)}$ a ser trabalhado desde 1996, após 15 anos nos deparamos ainda com fragilidades nas práticas pedagógicas dos professores nas diversas instituições de ensino. Retomamos a questão da prática do professor da educação básica em atender as recomendações das diretrizes do PCN, mas sabemos que possivelmente esta realidade seja resultado do processo de formação do professor. As questões relacionadas à sexualidade retoma a partir da década de 80 mediante as necessidades e preocupações em âmbito nacional de gestores, profissionais de saúde e educadores com o fenômeno da Aids ${ }^{(4-5)}$.

De fato, trabalhar conteúdos nesta temática e suas especificidades no ambiente escolar não é tarefa fácil, pois segundo estudos ${ }^{(13,22)}$ este tema é ainda considerado delicado e repleto de preconceitos e tabus, pois sofre influências externas de aspectos históricos, sociais e até religiosos e sobretudo o professor também tem suas concepções formalizadas e experiências pessoais bem sucedidas ou não, em relação a sexualidade.

Um estudo realizado com professores portugueses ${ }^{(3)}$ mostrou também dificuldades no trabalho da temática com os alunos e que não receberam conteúdos específicos durante sua formação, sugeriram a realização de cursos por meio de educação continuada com conteúdos complementares de temas específicos de acordo com a realidade de seus alunos. Outro estudo realizado com professores goianos ${ }^{(13)}$ na modalidade de pesquisa-ação mostrou resultados semelhantes ao estudo português sobre a não realização de trabalhos em salas de aula devido a deficiência na formação e completaram sobre a falta de experiência e conhecimento técnico-cientifico na temática, além de que a temática não constava nos PPP na ocasião da realização do estudo e também necessitavam se capacitar.

De acordo com os dados obtidos na presente investigação, faz-se necessário uma programação efetiva e parcerias entre as áreas da saúde, especialmente a Estratégia Saúde Família com apoio efetivo de profissionais para o suporte pedagógico nas escolas das áreas adstritas às Unidades Básicas de Saúde e área da educação com participação e apoio das Universidades com projetos de extensão e pesquisa com educação continuada na área da sexualidade. Fazendo referência às Universidades como importantes instituições formadoras e apoiadoras a outros níveis de ensino, é necessário refletir sobre o preparo técnico-científico também destes docentes que irão trabalhar a educação continuada e permanente na educação básica. Assim, a inclusão da Educação Sexual nos diferentes cursos de graduação é fundamental(2).

A permanência constante na escola faz com que o vínculo entre aluno e professor se fortaleça aliada da imagem de referência e exemplo por ser um grande detentor de informações e conhecimentos. Isso faz com que os adolescentes exponham suas dúvidas e compartilhem suas vivências com mais autonomia, naturalidade e liberdade.

A maioria dos professores informou que a temática sexualidade não constava no PPP da instituição pesquisada conforme preconiza os $\mathrm{PCN}^{(10)}$. O PCN almeja fomentar as instituições de ensino na elaboração de propostas, de forma flexível, a constar temas transversais nos currículos, ou seja, estar presente em todas as áreas do conhecimento, humanas, exatas e biológicas. Dentre os temas transversais destaca-se a educação sexual.

É necessário que os professores saibam da existência e finalidade do PCN devendo, portanto, participar da elaboração e atualização anual do PPP para então planejar seus conteúdos programáticos destacando a promoção da saúde sexual e reprodutiva dos alunos, na perspectiva da interdisciplinaridade e transversalidade ${ }^{(2,10)}$.

Estudos realizados com professores mostraram que esta realidade já foi incorporada pelos profissionais e que abordagens sobre sexualidade estão presentes nos conteúdos que ministram e que já constam formalmente nos PPP das instituições em que trabalham ${ }^{(4,23)}$.

Todo profissional que está diretamente envolvido com o processo de ensinar precisa de constante atualização de seus conhecimentos. Em se tratando de temas comportamentais, além de atualização dos conhecimentos, também se faz necessário que adotem metodologias de ensino mais participativas e dialógicas com utilização de recursos didáticos variados, podendo contribuir para uma prática pedagógica mais segura do professor no trabalho da temática. A capacitação profissional é fundamental para ampliar o aporte 
teórico, metodológico e assertividade dos educadores na implantação de atividades nesta área ${ }^{(24)}$. Há necessidade de atualização de temas com participação ativa dos jovens em oficinas interativas, participação de professores em eventos científicos, disponibilidade de materiais didáticos atualizados, além da parceria com profissionais de saúde para ampliação do conhecimento de professores na área da educação sexual.

\section{CONCLUSÃO}

Reflexões sobre conteúdos que envolvem a sexualidade no ambiente escolar certamente deslumbram muitos educadores, mas assusta outros. Contudo, vale ressaltar que o momento histórico em que vivemos se mostra favorável para discussões dessa temática na conjuntura da escola, considerando o grande contingente de jovens atualmente.

De acordo com os objetivos propostos verificamos que os resultados destacam a predominância da formação dos professores na área das ciências humanas; informações sobre o grande interesse dos alunos pela temática sexualidade; os professores não possuem experiência na temática que não está presente nos PPP conforme preconizado pelo PCN; relataram dificuldades no trabalho da temática; não receberam capacitações e, portanto necessitam se capacitar.

Há décadas que recomendações foram feitas pelas políticas educacionais, como o PCN, que possibilitasse ampliar discussões sobre educação sexual nas instituições de ensino brasileiras.

Os resultados do nosso estudo mostraram que há uma grande lacuna entre o ideal e o real, pois como identificado, ainda temas como a educação sexual, não constavam formalmente nos projetos pedagógicos das instituições de ensino pesquisadas.

Os resultados mostraram também que por meio dos interesses dos alunos na temática os professores podem suscitar discussões utilizando ferramentas como implementação de projetos e programas governamentais como SPE e PSE, que enfatizam a educação sexual como orientação para a vida, e prioriza a vulnerabilidade como eixo norteador. Há demandas juvenis a ser beneficiada com trabalhos de promoção da saúde sexual e reprodutiva, mas para isso faz-se necessário um trabalho mobilizador com foco na formação técnico-científica dos professores para o trabalho da educação sexual em todas as áreas do conhecimento, que possibilite a interação entre o pessoal e o profissional.

Sugerimos, portanto que parcerias envolvendo a Família, Escola, Profissionais da Área da Saúde e Instituições de Ensino Superior sejam estabelecidas no sentido de apoiar a Educação Básica na instrumentalização dos professores para trabalhos em Educação Sexual no contexto escolar. Mas, vislumbramos que para o sucesso do trabalho na instância da educação básica, há necessidade de reformulação curricular do ensino superior no sentido de incluir conteúdos em educação sexual nos diferentes cursos de graduação, considerando a transdisciplinaridade e a transversalidade.

Quando perguntado em qual disciplina seria abordado a temática, sobressaiu biologia na opinião dos professores. É realidade que os profissionais ainda planejam seus conteúdos de acordo com suas disciplinas. Pela preconização do PCN que assuntos/conteúdos transversais, como a educação sexual esteja contemplado nos PPP das instituições, os professores devem seguir assiduamente as normatizações previstas. Agindo assim certamente estarão rompendo com os discursos hegemônicos de que conteúdos sobre sexualidade não devem ser trabalhados apenas nas disciplinas das ciências biológicas, área ainda reconhecida pela forte tendência e vinculação aos aspectos biológicos do ser humano.

Após o término desta investigação, os resultados obtidos foram apresentados às coordenações locais das instituições pesquisadas e o grupo de pesquisadores se propôs a ofertar cursos de capacitação aos professores participantes, como proposta de projeto de extensão, na modalidade de oficinas interativas com conteúdos emergidos a partir dos interesses e necessidades dos professores.

Também é o desejo do grupo de pesquisadores realizarem novas investigações no sentido de conhecer outras instituições da rede estadual e municipal de ensino de diferentes regiões do município de Goiânia/GO e, assim, propor a educação continuada a outros profissionais que certamente apresentam necessidades e realidades bem parecidas com as do estudo. 


\section{REFERÊNCIAS}

1. Moizés JS, Bueno SMV. Compreensão sobre sexualidade e sexo nas escolas segundo professores do ensino fundamental. Rev Esc Enferm USP. 2010;44(1):205-12.

2. Silva RCP, Neto JM. Formação de professores e educadores para abordagem da educação sexual na escola: o que mostram as pesquisas. Ciência e Educação. 2006;12(2):185-197.

3. Ramiro L, Matos MG. Percepções de professores portugueses sobre educação sexual. Rev Saude Publica. 2008;42(4):684-92.

4. Barros SC, Ribeiro PRC. Educação para a sexualidade: uma questão transversal ou disciplinar no currículo escolar? Revista de Enseñanza de las Ciencias. 2012;11(1):164-187.

5. Boletim Epidemiológico - Aids e DST [Internet]. Ministério da Saúde (BR): Ministério da Saúde. Ano VIII, Nº 1, 2011 [cited 2013 fev 27]. Available from:

http://www.aids.gov.br/sites/default/files/anexos/publicacao/2 011/50652/boletim_aids_2011_final m_pdf_26659.pdf.

6. Furlani J. Sexos, sexualidades e gêneros: monstruosidades no currículo da educação sexual. Educação em revista. 2007;46:269285.

7. Oliveira DC, Pontes APM, Gomes AMT, Ribeiro MCM.

Conhecimentos e práticas de adolescentes acerca das DST/HIV/AIDS em duas escolas públicas municipais do Rio de Janeiro. Esc. Anna Nery. 2009;13(4)833-41.

8. Silva MAI, Mello DF, Carlos DM. O adolescente enquanto protagonista em atividades de educação em saúde no espaço escolar. Rev. Eletr. Enf. [Internet]. 2010 [acesso em: $20 \mathrm{dez}$ 2013];12(2):287-93. Disponível em: http://dx.doi.org/10.5216/ree.v12i2.5301.

9. Freitas KR, Dias SMZ. Percepções de adolescentes sobre sua sexualidade. Texto Contexto Enferm. 2010;19(2)351-7.

10. Ministério da Educação (BR). Parâmetros Curriculares

Nacionais: apresentação dos temas transversais, ética. Brasília (Brasil): MEC/SEF; 1997.

11. Albuquerque JG, Pinheiro PNC, Lopes MVO, Machado MFAS. Conhecimento deficiente acerca do HIV/AIDS em estudantes adolescentes: identificação de diagnóstico de enfermagem da NANDA. Rev. Eletr. Enf. [Internet]. 2012 [cited 2013 mai 19];14(1):104-11. Available from:

http://www.fen.ufg.br/revista/v14/n1/v14n1a12.htm.

12. Montardo J. A escola e a educação sexual. La Salle. Revista de educação, ciência e cultura. 2008;13(1):161-73.

13. Souza, MM; et al. Qualificação de professores do ensino básico para educação sexual por meio da pesquisa-ação. Cienc Cuid Saude. 2010;9(1):91-8.

14. Secretaria de Vigilância em Saúde, Ministério da Saúde. Programa Nacional de DST e Aids. Diretrizes para implantação do Projeto Saúde e Prevenção nas Escolas (SPE). Brasília (Brasil): Ministério da Saúde, 2006.

15. Ministério da Saúde; Ministério da Educação. Programa Saúde na Escola (PSE). Brasília (Brasil): Ministério da Saúde, 2008.

16. Duarte, EC. A Pesquisa Nacional de Saúde do Escolar (PeNSE, 2009) e a vigilância em saúde do escolar no Brasil: questões para reflexão. Cienc. Saúde Coletiva. 2010;15(Sup.2): 3021-23.

17. Malta et al. Saúde sexual dos adolescentes segundo a Pesquisa Nacional de Saúde dos Escolares. Rev Bras Epidemiol. 2011;14(1):147-56.

18. Ministério da Saúde. Secretaria de Atenção a Saúde. Saúde do Adolescente. Série B. Textos Básicos de Saúde. Brasília (Brasil): 2008.

19. Brasil. Lei $n^{\circ} 8.069$, de 13 de julho de 1990 . Dispõe sobre o Estatuto da Criança e do Adolescente e dá outras providências. Casa Civil- Subchefia para assuntos jurídicos. Diário Oficial da União (Brasília): 1990.
20. Ressel LB, Junges CF, Sehnem GD, Sanfelice C. A influência da família na vivência da sexualidade de mulheres adolescentes. Esc Anna Nery (impr.). 2011;15(2):245-250.

21. Reis $M H$, Vilar D. A implementação da educação sexual na escola: Atitudes dos professores. Análise Psicológica. 2004;4(12):737-745.

22. Song AV, Halpern-Felsher BL. Predictive Relationship Between Adolescent Oral and Vaginal Sex - Results From a Prospective, Longitudinal Study. Arch Pediatr Adolesc Med. [Internet]. 2010. [Available from:2013 Mai 25]; nov; (1):214-24. http://scielo/archpediatrics/pdf/n1.

23. Silva MP, Carvalho WLP. O desenvolvimento do conhecimento pedagógico do conteúdo de sexualidade na vivência de professoras. Ciência e Educação. 2005;11(1):73-82. 24. Biscolli C, Favarão NRL, Feiten RH, Souza ACP, Perpétuo CL. Sexualidade em sala de aula: um estudo da produção de sentidos. Arq. Ciênc. Saúde Unipar, Umuarama. 2005;9(1):47-55.

Artigo recebido em 05/09/2012.

Aprovado para publicação em 14/05/2013.

Artigo publicado em 31/12/2013. 